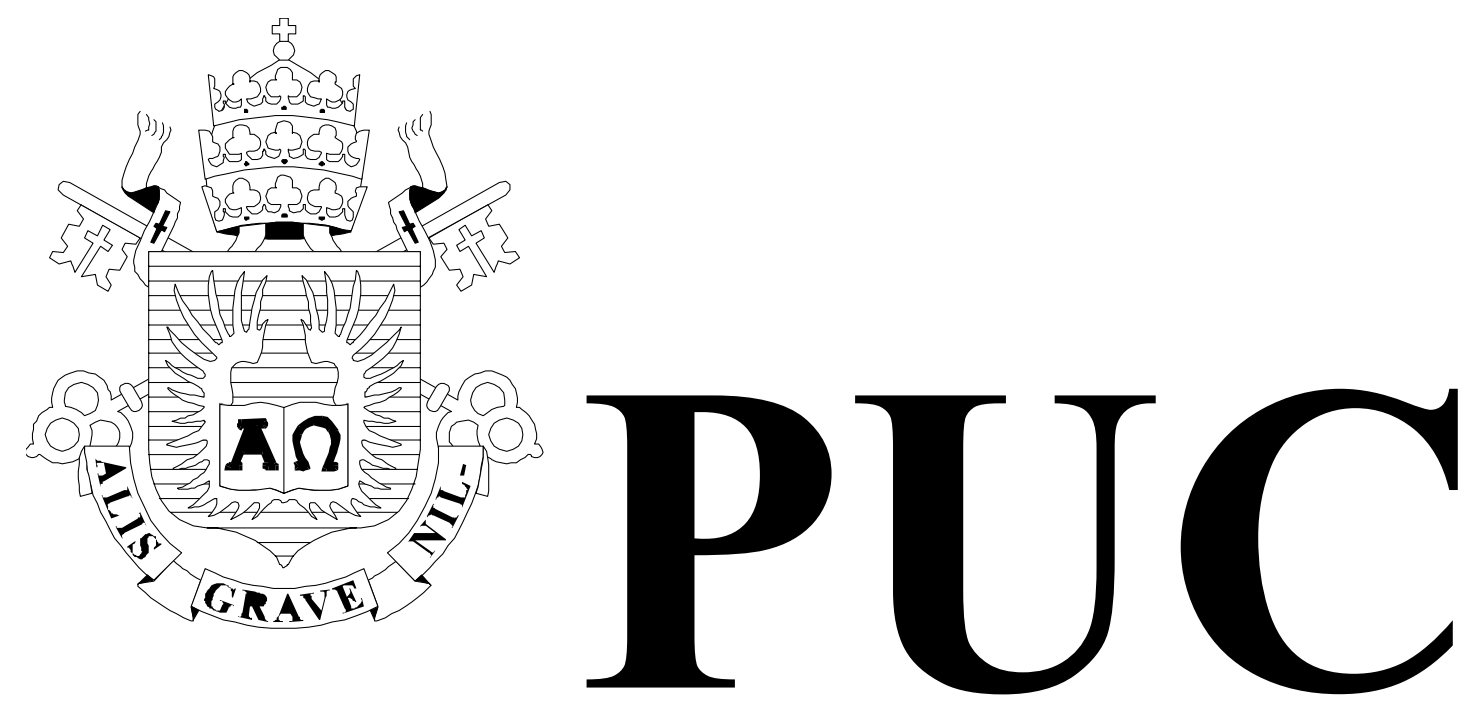

ISSN 0103-9741

Monografiasem Ciência da Computação $n^{\circ} 57 / 03$

Finding Solutions for Effective Collaboration in a Heterogeneous Industrial Scenario

Isma el Humberto Ferreira dos Santos

Alberto Barbosa Raposo

Marcelo Gattass

Departamento de Informática 
PUC RIO - DEPARTAMENTO DE INFORMÁTICA

ISSN 0103-9741

Monografiasem Ciência da Computação, № 57/03

Editor: CarlosJ. P. Lucena

Dezembro, 2003

Finding Solutions for Effective Collaboration in a Heterogeneous Industrial Scenario

Ismael Humberto Ferreira dos Santos

Alberto Barbosa Raposo

Marcelo Gattass 


\title{
Finding Solutions for Effective Collaboration in a Heterogeneous Industrial Scenario
}

\author{
Ismael H. F. dos Santos ${ }^{1,2}$, Alberto B. Raposo ${ }^{1}$, Marcelo Gattass ${ }^{1}$ \\ ${ }^{1}$ Computer Graphics Group (Tecgraf) \\ Computer Science Dept. - Catholic University of Rio de Janeiro (PUC-Rio) - Brazil \\ ${ }^{2}$ CENPES - PETROBRAS Research Center - Brazil \\ \{ismael, abraposo,gattass\}@tecgraf.puc-rio.br \\ PUC-Rio.Inf.MCC57/03 December, 2003
}

\section{Resumo}

Este artigo apresenta um modelo para a categorização de colaboração apoiada por computador em vários níveis, de acordo com o suporte fornecido à comunicação, à coordenação, à cooperação e à interoperabilidade. $\mathrm{O}$ modelo apresentado é útil para a definição do nível de colaboração desejado em cenários industriais ou do nível que se pode atingir com a tecnologia e os recursos existentes. Essas idéias são ilustradas por meio de ferramentas colaborativas da área de Computação Gráfica sendo desenvolvidas para uma grande empresa da área de petróleo.

Palavras-Chave: CSCW, Groupware, Realidade Virtual, Computação Gráfica

\begin{abstract}
This work introduces a model that categorizes computer-supported collaboration in different levels, according to its support for communication, coordination, cooperation and interoperability. This model is useful for defining the level of collaboration needed in real industrial scenarios or the level that is possible to achieve with the available technology, resources and working culture. These ideas are illustrated by collaborative tools in the field of Computer Graphics being developed for a large oil \& gas company.
\end{abstract}

Key-Words: CSCW, Groupware, Virtual Reality, Computer Graphics 


\section{Introduction}

The work presented in this paper is motivated by the necessity of effective solutions for collaboration of PETROBRAS, a large Brazilian governmental oil \& gas company.

This necessity is especially acute in the field of Computer Graphics, whose techniques such as scientific visualization and three-dimensional geometric modeling have been revolutionizing the oil industry, especially in the exploration and production field (E\&P). The possibility of visualizing and manipulating virtual "earth models" in the computer has completely changed the professional's way of working, notably for the geologists, geophysicists and engineers [12].

Recently the arrival of immersive visualization environments, Virtual Reality environments (VEs), with integrated audio, videoconferencing systems, large display walls, stereo graphics projection and haptic peripherals, is pushing the limits in teamwork activities especially in Reservoir and Offshore Engineering. In the former area large research is being conducted to create better techniques to produce reliable 3D models for the target reservoir. Usually the specialists are forced to use many different software, commercial and homemade, to accomplish their task in a reasonable time. In the second field the research involves specialists from different areas. The activities include naval and structural projects of oil production plants and the associated mooring systems, computational fluid dynamics simulations for the stability of those systems, and project and planning the installation of the submarine arrangement of the deep water equipments.

The scenario described above is highly heterogeneous, being composed of not only geographically distributed teams, but also teams of specialists in different areas using different software.

An additional difficulty, specially acute in Offshore Engineering is that, although the specialists deal with the same artifacts (platforms, production risers, mooring systems, etc.), they usually have different data representations for those objects according to the needs of each application. For example, in structural and naval engineering the models usually have dense polygonal meshes, with a few objects representing the outline of the artifacts, suitable for static and dynamic stability studies with some numerical methods such as finite and boundary element methods. In CAD/CAE the models usually have objects with coarse grid meshes suitable for giving a reasonable visual representation, but the problem is that all the objects that comprises the artifact should be represented yielding huge models. For real time visualization those models are almost intractable and, even today, represents a research challenge for computer graphics [10]. A typical CAD/CAE platform model usually has 3 million triangles while the mooring system has approximately 1 million triangles.

Another problem of Offshore Engineering is that projects in this field are segmented due to their huge complexity. They are normally divided into independent subprojects where each one deals with an abstract or equivalent representation of the others. In order to accomplish collaboration and interoperability between those subprojects, a software-based interface is required, which to the best of our knowledge is not available today.

Our approach to tackle those problems is to develop a collaboration framework that integrates users located at different places using their respective applications. Moreover, the framework should also provide a full interoperable scenario, allowing not only the use of different software but also a transparent flow of information among the different users with their distinct models and projects.

In order to achieve this solution we propose a hierarchy of collaborative scenarios to depict the different levels of collaboration that might exist in a computer organization. This hierarchy paves the way for achieving our ultimate collaboration and interoperability goal. Such hierarchy is used for planning the successive phases of the AmbVirt project, which is being conducted by PETROBRAS and Tecgraf/PUC-Rio for developing Collaborative Virtual Environments for downstream and upstream applications.

In the following section we define collaboration models that constitute the theoretical basis of our hierarchy. The hierarchy and their collaborative scenarios are presented in Section 3. Then we give a brief overview of AmbVirt project in Section 4, and finish with the conclusions. 


\section{Collaboration Models}

The collaboration levels that are going to be presented in the next section are based on two models that analyze collaborative activities in several aspects: the 3C model (communication, coordination, cooperation) and the model that divides groupware in collaboration-aware and collaboration-unaware applications.

\subsection{The 3C Model}

In order to work collaboratively, people need to share information (communication). Communication, although vital, is not enough; "it takes shared space to create shared understandings" [16]. This notion of shared workspace (including user awareness, shared objects, etc.) is called cooperation. To cooperate, however, people need to work harmoniously, avoiding conflicting or repetitive actions (coordination). These aspects (communication, cooperation and coordination) constitute a threesome frequently associated with collaboration [5], [8].

Collaborative applications, according to the 3C model, are composed of tools providing one or more of the three functionalities described above.

Another central aspect of the 3C model is the notion of user awareness, which is defined as the way users perceive other participants of the collaboration and what they are doing, without direct communication between them [4]. Awareness elements are essential for the collaboration flow, because they enable the user to build his/her own work context and to coordinate his/her activities with those of the others. Therefore, user awareness may be considered the fourth element of the 3C model, which is deeply related to communication, coordination and cooperation.

\subsection{Collaboration-aware and Collaboration-unaware Applications}

The applications available for computer-supported collaboration can be divided into two categories, depending on how the support for collaboration is related to the application implementation: they can be collaboration-aware or collaboration-unaware applications [14].

Collaboration-unaware applications are originally developed to be single user applications, but may be used collaboratively by means of an external support system. This external support system may be an application sharing system, such as Microsoft NetMeeting, or a GUI event multiplexing system. The difference is that in the first case, only one user is running the application whose windows' contents are broadcast to all connected users. All users' inputs are gathered and serialized, to be received by the application as if it were used by a single user. In the second case, all users are running an instance of the application, with a special layer between the application's GUI and its event handler. This layer broadcasts all GUI events to connected users and interprets all received remote events as if they were generated by the local user. In both cases the applications do not explicitly support collaboration; they are implemented as single user applications [18].

Collaboration-aware applications, on the other hand, are specially developed for the collaborative work. They constitute distributed systems (centralized or replicated) that are aware of the communication channels among the distributed instances of the application, the information exchange among them, the number of connected users and their role in the collaboration, the coordination policies, among other aspects of communication, coordination and cooperation. 


\section{Collaboration Scenarios}

The process of adopting collaboration tools in organizations is sometimes considered as a "dual process of both adapting the organization of work to the conditions of the tool, and adapting the tool to meet this organization of work" [2]. Although we do not disagree with this consideration, it is our belief that the chances of success are immensely higher if we adopt collaboration solutions that meets the actual organization of work in the company. Therefore, we used the models described in the previous section as a basis to create a number of collaboration scenarios that are viable to the various necessities and restrictions discussed in the first section.

We have been developing a collaboration infrastructure composed of 5 levels, where each level incrementally incorporates some degree of collaboration, ranging from level 0 with no support for collaboration, to level 5 with a fully interoperable collaborative scenario (Figure 1).

At level 0 we do not have any specific computer support for collaboration and the mechanisms used are basically the telephone for communication and any suite of network protocols (with support for file transfer, terminal emulation, etc.) to exchange information among users.

At level 1, called video-based awareness, a higher degree of communication is achieved with integrated audio and videoconferencing systems (communication). More than a simple desktop videoconferencing system it is important to share the workspace (cooperation), i.e., the participants should be able to see, by video transmission, the data being visualized by their peers. In this scenario the concept of awareness is introduced as a mechanism to reinforce communication and cooperation among the users. At this level we do not have a "complete" collaboration scenario, since the remote user is not able to interact with the application. However, the notion of shared workspace is present, since it is possible, for example, to exchange information pointing to a specific part in the artifact's working model.

At level 2, software-based awareness, a higher level of cooperation is provided by the application as well as some level of coordination, which is implemented to give the user the notion of what is going on the other side. Typical applications at this level use the client-server paradigm to exchange information among the server and the clients. The server is responsible for transmitting the workspace to the clients, instead of only being transmitted by video. At this level collaboration is still limited, in the sense that the clients cannot directly interact with the broadcasted workspace. However, the shared workspace is even more acquainted to the user, since it is present in his own computer, not as streaming video.

At level 3, collaboration-unaware application, the support for collaboration is given for applications that are originally developed to be single user applications. Once the applications do not have explicit support for collaboration, this support is provided by an external system. At this level collaboration is bi-directional, in the sense that all users may interact in the shared workspace.

The difference between level 3 and level 4, collaboration-aware application, is that the support for collaboration in the latter is provided by distributed applications specially developed for that purpose. Although offering better collaboration capabilities than the previous levels, level 4 incurs in longer and more expensive development efforts, which are not always possible.

At level 5, interoperable collaborative application, the collaboration support provided by level 3 and/or level 4 is complemented with a framework for interoperability among different applications. The objective of this framework is to allow that the shared artifacts from the various users transparently move throughout the multi-application workspace. In this scenario, users should be able to use different software that deal with several data formats and their different representations, maintaining the representation on the various applications synchronized and consistent.

It is important to mention that the lower levels, 1 and 2, though having poorer collaborative resources, are easier to implement and, in some cases, are the only viable solutions due to the available infrastructure and/or budget constraints. Moreover, in some cases where the most important tools used in the environment are commercial software with non-extensible functionalities it is not possible to reach the higher collaboration levels, which require intrusive interventions in the software. 


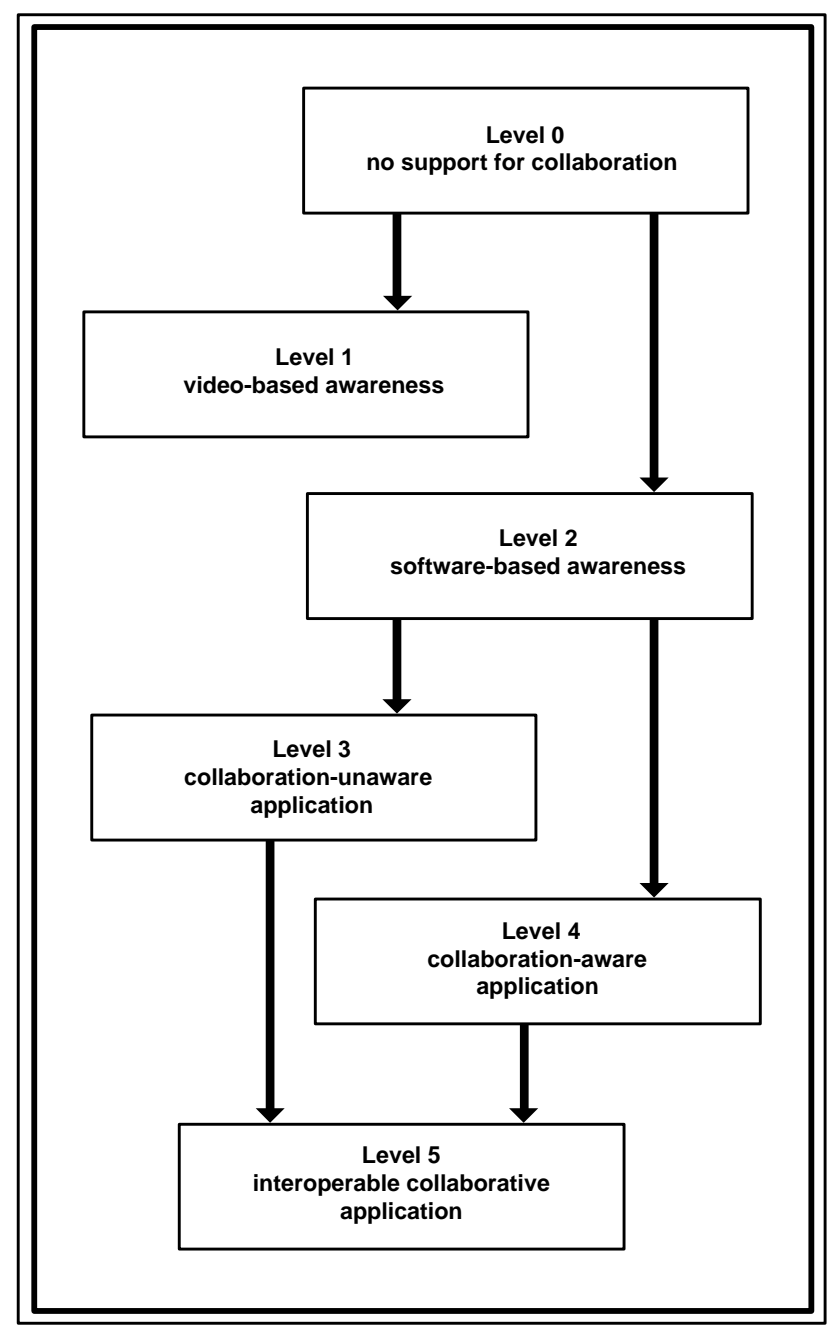

Figure 1. Hierarchy of collaborative scenarios.

\section{The AmbVirt Project}

Collaborative Virtual Environments (CVEs) appears as a natural evolution of the VE and the advances achieved in fields such as computer networks and distributed systems [17]. CVEs can be seen as the result of a convergence of research interests within the Virtual Reality and Computer-Supported Cooperative Work. The adoption of CVEs by nation wide companies like PETROBRAS is of vital importance because it allows joining specialists that are far away in meetings or team training reducing the expenses with voyages [7]. The difficulties for adopting a CVE as a general visualization tool come from the fact that some of the activities in PETROBRAS are done with commercial software, which makes difficult the integration of those with a CVE.

During the last few years PETROBRAS has been investing a considerable amount of money in the construction of visualization environments, spread among several branches in the country, composed of rooms equipped with large display and stereo projectors for downstream and upstream visual applications.

In order to find effective solutions for collaboration and interoperability among those environments, PETROBRAS and FINEP (a project financing agency of Brazilian Ministry of Science and Technology) are sponsoring a project called AmbVirt, which is being conducted by Tecgraf/PUC-Rio and CENPES, 
PETROBRAS Research Center. This project is divided into four research areas with the goal to achieve the highest collaboration level by the conjunction of efforts (Figure 2).

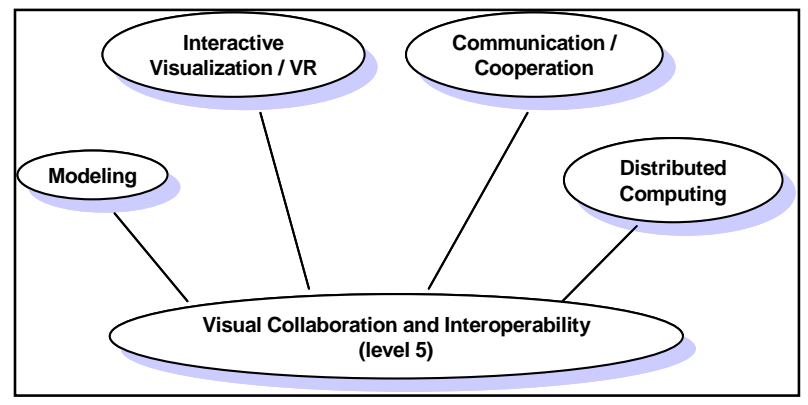

Figure 2. Research areas of AmbVirt project.

The area of modeling is responsible to what may be considered the input of the collaborative environment, i.e., the models for the graphical data. The efforts in this area are related to the study of interoperability and conversion among the several types of models used, as a means to achieve the required interoperability at level 5. Examples of tools that already provide some degree of interoperability are 3D ACIS InterOp [1] and OpenSpirit [13]. The former provides interoperability components that simplify and accelerate 3D data transfer between disparate formats and platforms. OpenSpirit is a platform independent plug-and-play CORBA-based framework that allows applications from a variety of vendors in the field of petroleum to interoperate.

The area of visualization acts on the other extreme, i.e., the output. The efforts in this area are related to the definition of a common virtual reality environment and collaborative visualization techniques. Some of the tools explored in this area are the VRJuggler [19] and the DVRL [6]. VRJuggler is a C++ class library that is used as a framework for virtual reality application development. The DVRL (Distributed Virtual Reality Library) is a homemade library to synchronize multiple displays in distributed environments.

The other two areas may be viewed as providers of the support needed in the collaborative scenarios. The area of communication and cooperation deals with CSCW and groupware issues, aiming to provide the appropriate framework for collaboration. The distributed computing area provides the lower level support needed with the implementation of services and distributed components required by the collaboration framework.

In order to immediately introduce collaboration in the work scenario, the first step of the project has been the development of a customized videoconferencing tool connecting the visualization rooms. This tool, called CSVtool (Collaboration between Visualization Rooms), reaches the level 1 of collaboration, since it builds a scenario where the cameras located in the rooms send video streams to other connected rooms. There is at least a camera focusing the large display, plus some additional ones focusing the audience and/or the users' desktops.

Figure 3 sketches out a scenario where two rooms, each one with two projectors, are collaborating. In this figure, room A has a camera sending its local data to be visualized in room B, while room B has a camera focusing on the audience, whose image is transmitted to room A. More cameras and more rooms may be added to the scenario. Figure 4 shows a shot of the first CSVtool prototype, where a remote desktop user is receiving videos from the projections of two visualization rooms. Since data are being projected on each room screens, users may enrich their interaction capabilities by, for example, pointing to specific aspects in the visualizations, creating the notion of a shared workspace.

Currently a level 2 application is also being developed to work in a real environment. The objective of this application, called Wlog (Well Log) is to allow the specialists (geologists, geophysicists and reservoir engineers) located in visualization rooms at the headquarter to accompany, in real time, the drilling process through the broadcasting of the data collected at the well [3]. During the drilling the specialists can give instructions to guide the operator in order to optimize the process of drilling. 


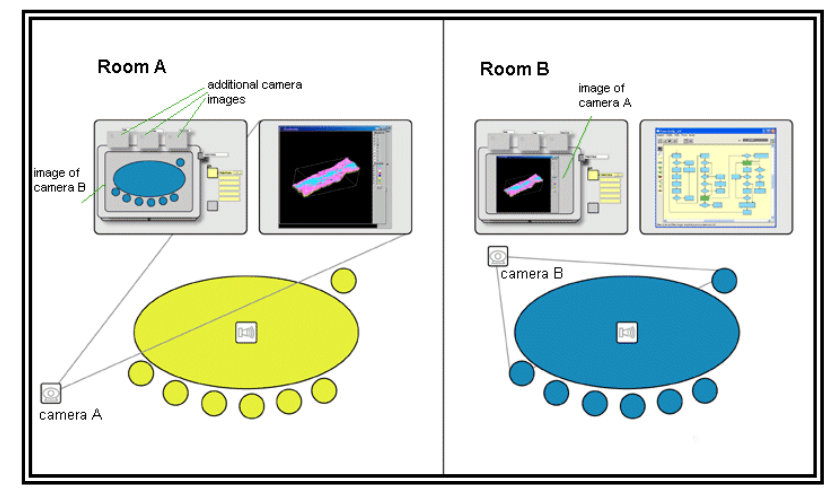

Figure 3. Sketch of a video-based awareness (level 1) scenario.

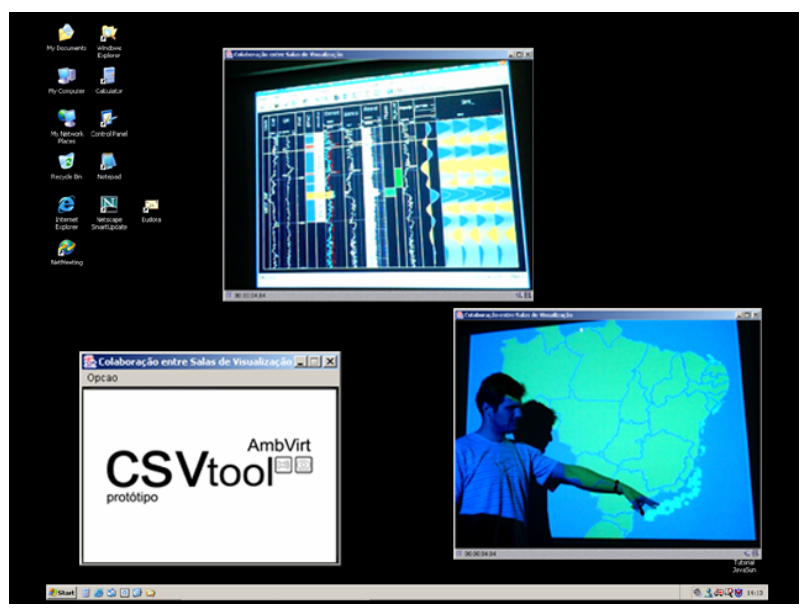

Figure 4. Shot of a session using the CSVtool.

The application is constructed using the gOcad [9] software, a geological modeler used to model the subsurface geometry and earth properties, and Lua [11] which is a powerful light-weight programming language designed for extending applications, developed at Tecgraf/PUC-Rio. This application is considered to be in level 2 because the clients only receive the well data from the server located at the well location, and update the replicated model being visualized at the visualization room without being able to interact with the visualization process.

At level 3, a collaborative plugin is being developed for gOcad [15]. The objective of this plugin is to allow the collaborative building of earth models by specialists located at different places.

For the level 4, some homemade applications in the area of Reservoir and Offshore Engineering are going to be transformed in collaborative applications using the support of the collaboration framework being developed and validated by the previous experiences.

After having a good experience with solutions built at levels 3 and 4, we plan to extend the applications developed in those levels in order to allow them to interoperate. Once working in a fully interoperable environment we will be able to solve some of the aforementioned problems. The shared artifacts will be manipulated transparently by the different software and the transitions between each representation will be 
fulfilled by the interoperable framework. Moreover all the projects comprising a large enterprise will be able to interact in a common CVE reducing the risks, the time and the cost of the projects.

\section{Conclusion}

This paper presented an approach based on the definition of collaboration levels that may drive the introduction of computer-supported collaborative practices in organizations. The approach is specially suited for highly heterogeneous scenarios involving different software, specialists in different areas with particular information models, and technological or cultural restrictions to the implementation of a fully collaborative environment. Although general, the approach was presented by the particular situation of implementing a collaborative scenario for Computer Graphics applications in an oil \& gas company.

At the lower collaboration levels, the proposed approach enables a rapid and relatively inexpensive solution for introducing simple and unobtrusive collaborative scenarios. At the higher level the approach envisages a fully collaborative and interoperable scenario, embracing several issues related to the heterogeneity of large organizations.

As next steps of this work, we plan to continue evaluating the approach based on the experience with the developed collaborative tools in the real scenario.

\section{Acknowledgement}

The authors would like to thank PETROBRAS and FINEP, the sponsors of AmbVirt project, and also Tecgraf/PUC-Rio and CENPES for the expressive support. Tecgraf/PUC-Rio is a laboratory mainly funded by PETROBRAS. Our thanks also go to all the group working in the AmbVirt project, specially Dr Alvaro Maia and Dr Anelise Lara, managers of the research groups supporting the project at CENPES.

\section{References}

[1] Spatial Corp., “3D IterOp Components”, http://www.spatial.com, 2002.

[2] J. E. Bardram, “Organizational Prototyping: Adopting CSCW Applications in Organisations”, In G. Mark et al. (organizers), Position Papers of the CSCW 96 Workshop "Introducing groupware in organizations: What leads to successes and failures?”, 1996.

[3] J. L. E. Campos, "Real-Time Well Drilling Monitoring using gOcad”, 22nd Gocad Meeting, Nancy, France, 2002.

[4] P. Dourish, and V. Bellotti, “Awareness and Coordination in Shared Workspaces”, Proc. of the Conf. on Computer Supported Cooperative Work (CSCW’92), 1992, pp. 107-114.

[5] C. A. Ellis, S. J. Gibbs, and G. L. Rein, “Groupware: Some Issues and Experiences”, Communications of the ACM, 34(1): 38-58, 1991.

[6] A. Ferreira, R. Cerqueira, W. Celes, and M. Gattass, "Multiple Display Viewing Architecture for Virtual Environments over Heterogeneous Network”, XII Brazilian Symposium on Computer Graphics and Image Processing, 1999, pp. 83-92.

[7] J. A. Friesen, and T.D. Tarman, "Remote High-Performance Visualization and Collaboration”, IEEE Computer Graphics and Applications, 20(4): 45-49, 2000.

[8] H. Fuks, C. Laufer, R. Cohen, and M. Blois, "Communication, Coordination and Cooperation in Distance Education”, V Americas Conference on Information Systems (AMCIS'99), 1999, pp. 130-132. 
[9] “gOcad - consortium”, http://www.ensg.u-nancy.fr/GOCAD, 2002.

[10] M. Hofmam, M. Gattass, and W. Celes, "Organization of Large Engineering Models for Real-Time Walk-Through Rendering”, accepted for ICES’02 International Conference on Computational Engineering \& Sciences, 2002.

[11] “The programming language Lua”, http://www.lua.org, 2002.

[12] “Crucial Issues”, Oil \& Gas Executive Report, 2(2): 13-16, 1999.

[13] The OpenSpirit Corp., “OpenSpirit”, http://www.openspirit.com, 2002.

[14] W. Reinhard et al., “CSCW Tools: Concepts and Architectures”, IEEE Computer, 27(5): 28-36, 1994.

[15] L. P. Reis and J. C. Paul,, "Toward collaborative geomodeling”, 21st Gocad Meeting, 2001, http://www.ensg.u-nancy.fr/GOCAD/meetings/Nancy2001/index.html.

[16] M. Schrage, No more Teams! Mastering the Dynamics of Creative Collaboration, Currency Doubleday, New York, 1995.

[17] S. Singhal, and M. Zyda, Networked Virtual Environments: Design and Implementation, ACM Press, 1999.

[18] D. A. Tietze, “A Framework for Developing Component-based Co-operative Applications”, Dr.-Ing. Dissertation, Technischen Univerität Darmstadt, 2001.

[19] “VRJuggler - Open Source Virtual Reality”, http://www.vrjuggler.org, 2002. 